\title{
Damage Identification of Bridge Based on Modal Flexibility and Neural Network Improved by Particle Swarm Optimization
}

\author{
Hanbing Liu, Gang Song, Yubo Jiao, Peng Zhang, and Xianqiang Wang \\ College of Transportation, Jilin University, No. 5988 Renmin Street, Changchun 130025, China \\ Correspondence should be addressed to Yubo Jiao; jiaoyubo86@163.com
}

Received 3 June 2014; Accepted 20 July 2014; Published 14 August 2014

Academic Editor: Bin Yu

Copyright ( 2014 Hanbing Liu et al. This is an open access article distributed under the Creative Commons Attribution License, which permits unrestricted use, distribution, and reproduction in any medium, provided the original work is properly cited.

\begin{abstract}
An approach to identify damage of bridge utilizing modal flexibility and neural network optimized by particle swarm optimization (PSO) is presented. The method consists of two stages; modal flexibility indices are applied to damage localizing and neural network optimized by PSO is used to identify the damage severity. Numerical simulation of simply supported bridge is presented to demonstrate feasibility of the proposed method, while comparative analysis with traditional BP network is for its superiority. The results indicate that curvature of flexibility changes can identify damages with both single and multiple locations. The optimization of bias and weight for neural network by fitness function of PSO algorithm can realize favorable damage severity identification and possesses more satisfactory accuracy than traditional BP network.
\end{abstract}

\section{Introduction}

As important components of transportation infrastructure, bridges are essential for normal operation of transportation system. However, their loading capacities are threatened by external environment (such as vehicles and temperature), which reduces the level of security service. Therefore, it is necessary to periodically monitor the health status of structure [1]. Damage identification is one of the major challenges in bridge health monitoring. It can help to evaluate the safety condition, prevent catastrophic collapse, and also provide information for maintenance [2].

The damages of bridge can be defined as any deviation in structure's original geometric and material properties [3]. The reasons include reduction of structural stiffness, material cracks, and fatigue failure. A number of methods have been proposed in the past two decades to detect and assess the damage condition of bridge. Nondestructive methods such as ultrasonic waves, $\mathrm{X}$-ray, and stress waves have been widely applied in practice considering their convenience and simplicity. Most of these methods, however, rely on a presumption of the determination of suspected damage regions and are restricted for local detection. When applied to large structures, these methods are time-consuming and costly $[4,5]$.
Vibration-based damage identification methods are active in this research area, which, as compared to local nondestructive methods, does not require the a priori knowledge of damage locations. Damage can be detected based on changes in natural frequencies and mode shapes, which can be regarded as global methods. The techniques are based on the theoretical basis where damages alter the physical properties of bridge (e.g., mass, stiffness, and damping). Furthermore, the dynamic characteristics (e.g., mode shape, frequency, and damping ratios) will change. Therefore, damage location and severity can be assessed by analyzing the structure's dynamic characteristics [6-8].

Natural frequencies are widely used as damage indicators in the early stage of dynamic-based damage detection as they are easy to obtain. However, they are verified to be insensitive to damage and susceptible to environmental changes $[3,8]$. Mode shapes contain more information of damage location and are more suitable for damage identification than natural frequencies. However, it is difficult to realize the damage localizing that relies only on mode shapes data [6]. Modal flexibility has shown itself to be a promising damage indicator due to its high sensitivity to damage, because it contains the information of both natural frequency and mode shape. Pandey and Biswas [9] proposed a damage identification 
approach using changes in modal flexibility for the first time. In reality, it is impractical to identify all of the modes. However, the flexibility matrix can be accurately estimated based on the first few modes because it is inversely proportional to the squares of natural frequencies. Li et al. [10] applied the flexibility approach to damage identification of cantilevertype structures. Stutz et al. [11] presented a flexibility-based continuum damage detection method. Reynders and de Roeck [12] proposed a local flexibility-based approach which allowed determining the local stiffness variations directly from modal properties. It was verified by numerical simulation of damaged isostatic and hyperstatic beam and also experiments of a reinforced concrete beam. Catbas et al. [13] adopted modal flexibility for identifying structural behavior after damage which could be evaluated by inspecting the deflected shapes.

Most of dynamic-based damage identification methods can be used to identify damage presence and locations. But it is difficult to assess the damage severity. Furthermore, the computational time and costs are other challenges in damage detection. The applications of different computational intelligence approaches such as artificial neural networks (ANNs), genetic algorithm (GA), and particle swarm optimization (PSO) have been proved to be effective in damage severity assessment and improving the computational efficiency [14$16]$. ANNs $[17,18]$ are information processing systems which mimic the network structure of actual human brain. Mehrjoo et al. [19] presented a method for assessing the damage intensities of joints for truss bridges using BP network. Natural frequencies and mode shapes were adopted as input variables. Numerical simulation was used to demonstrate the accuracy and efficiency. Min et al. [17] proposed an innovative ANNsbased pattern analysis tool which can identify damagesensitive frequency and realize the identification of damage type and severity. However, ANNs have some drawbacks, such as the low convergence speed, overfitting, and locally optimal solutions [20].

PSO is a population based stochastic optimization technique developed by Eberhart and Kennedy in 1995, which is a new swarm intelligence technique inspired by social behavior of bird flocking or fish schooling [21]. Comparing with other soft computing tools, PSO is more efficient and requires fewer number of function evaluations, which leads to better or the same quality of results [22]. In past several years, PSO has been successfully applied in many research and application areas. Sharafi and Elmekkawy [23] proposed a novel approach for optimal design of hybrid renewable energy systems and PSO-simulation was used to solve the multiobjective optimization problem. Chen et al. [24] developed a method for reliability-based design of composite structures which was based on PSO and finite element analysis. Examples revealed that the proposed method had good stability.

In this paper, a two-stage damage identification method is proposed. Firstly, the damage location can be detected through modal flexibility indices. Secondly, ANNs are optimized by PSO for damage severity identification and the modal flexibility changes are treated as input variables. Numerical simulation is used to verify its feasibility of the proposed method.

\section{Theoretical Background}

2.1. Modal Flexibility. For a bridge system with $n$ degrees of freedom, the flexibility matrix $F$ can be calculated by [25]

$$
F=\left[f_{i, j}\right]=\sum_{i=1}^{n} \frac{\phi_{i} \phi_{i}^{T}}{\omega_{i}^{2}},
$$

where $\omega_{i}$ is the $i$ th natural frequency, $\phi_{i}$ is the $i$ th mass normalized mode shape, and $f_{i, j}$ is the modal flexibility component.

As can be seen from (1), the modal contribution to the flexibility matrix decreases rapidly as the frequency increases. Therefore, it can be obtained by the first few lower modes in practice.

The flexibility change matrix $\Delta F$ due to damage can be obtained by

$$
\Delta F=F_{D}-F_{I},
$$

where $F_{D}$ and $F_{I}$ are flexibility matrices for damaged and intact structure, respectively.

For each DOF $j$, the maximum absolute value of elements in $j$ th column of $\Delta F$ can be acquired as

$$
\delta_{j}=\max _{i}\left|\Delta f_{i, j}\right| .
$$

$\delta_{j}$ can be used to locate damage for bridge structures.

The curvature of flexibility changes can be calculated through second order central difference, which can be expressed as

$$
M F C_{i}=\frac{\delta_{i-1}-2 \delta_{i}+\delta_{i+1}}{\Delta l^{2}}
$$

where $\Delta l$ is the length of structural element.

\subsection{Neural Network Optimized by PSO}

2.2.1. Artificial Neural Networks. ANNs can process complex logic operations and achieve nonlinear mapping adaptively through learning. The process contains the forward propagation and back propagation. Neural network with three layers is shown in Figure 1.

A three-layer network typically consists of an input layer, a hidden layer, and an output layer. Each neuron possesses a bias, a transfer function, and an output. Neurons in both previous and subsequent layers are connected with each other. The most widely used learning algorithm is back propagation (BP) algorithm, which is a supervised learning style. In this algorithm, data are transferred forward, while errors are backward. In the process of forward, input information can be calculated through the hidden layers and finally delivered to the output layers. If the calculated results are not consistent with the expected one, then back propagation begins. Weights and bias are adjusted by prediction errors. Thereby, output results continue close to the expected ones.

2.2.2. Particle Swarm Optimization. In PSO algorithm, each solution is based on concept of the bird flock and is referred 


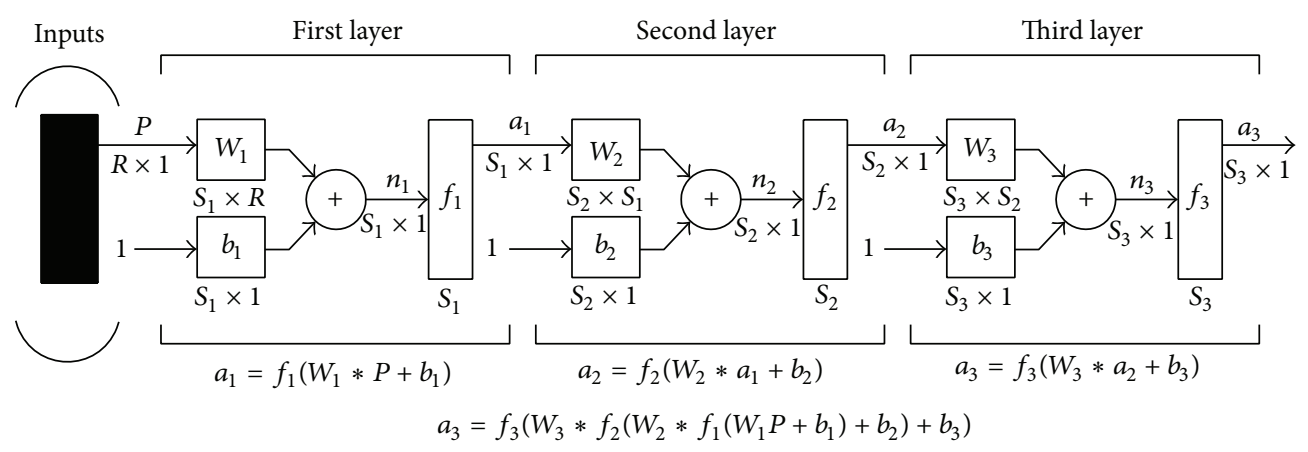

FIGURE 1: Structural diagram of ANNs.

to as a particle. In this framework the birds, besides having individual intelligence, also develop some social behavior and coordinate their movement towards a destination $[22,23,26$, 27].

Overall scale of particle swarm is assumed to be $n$, and the vector of coordinate position for each particle in $D$-dimension space can be expressed by

$$
\vec{X}_{i}=\left(x_{i 1}, x_{i 2}, \ldots, x_{i d}, \ldots, x_{i D}\right) \text {. }
$$

The velocity vector can be denoted by

$$
\vec{V}_{i}=\left(v_{i 1}, v_{i 2}, \ldots, v_{i d}, \ldots, v_{i D}\right) .
$$

And the best position of individual particle is

$$
\overrightarrow{P_{i}}=\left(p_{i 1}, p_{i 2}, \ldots, p_{i d}, \ldots, p_{i D}\right)
$$

While the best position for particles swarm can be expressed by

$$
\overrightarrow{P_{g}}=\left(p_{g 1}, p_{g 2}, \ldots, p_{g d}, \ldots, p_{g D}\right)
$$

The iteration of best position for individual particle can be realized by

$$
\begin{array}{cc}
p_{i, t+1}^{d}=x_{i, t+1}^{d}, & f\left(X_{i, t+1}\right)<f\left(P_{i, t}\right), \\
p_{i, t+1}^{d}=p_{i, t}^{d}, & f\left(X_{i, t+1}\right) \geq f\left(P_{i, t}\right) .
\end{array}
$$

The optimal position for particle swarm is the best one for individual particle, and the iteration of velocity and position can be calculated by

$$
\begin{gathered}
v_{i, t+1}^{d}=v_{i, t+1}^{d}+c_{1} * \operatorname{rand} *\left(p_{i, t}^{d}-x_{i, t+1}^{d}\right) \\
+c_{2} * \operatorname{rand} *\left(p_{g, t}^{d}-x_{i, t}^{d}\right), \\
x_{i, t+1}^{d}=x_{i, t}^{d}+x_{i, t+1}^{d} .
\end{gathered}
$$

The calculation flowchart for PSO algorithm is shown in Figure 2.

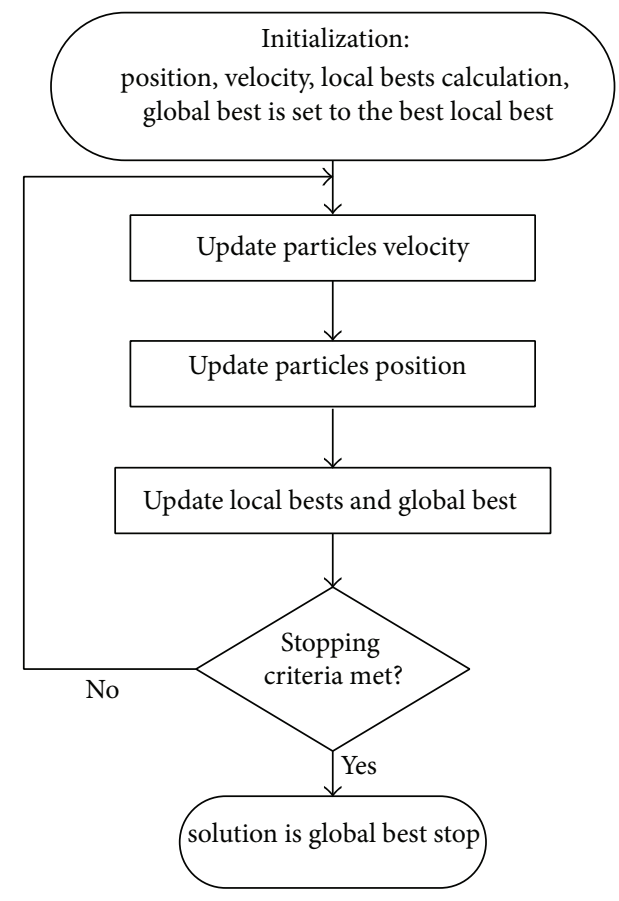

FIGURE 2: PSO algorithm flowchart.

2.3. PSO-BP Hybrid Algorithm. ANNs suffer from slow convergence and getting suck in local minima. Therefore, PSO is used to optimize ANNs in order to achieve the combination between global optimization of PSO and instructive search of ANNs. The hybrid algorithm can not only avoid the local convergence phenomenon but also improve the performance of networks [28]. The bias and weight can be adjusted through the fitness function of PSO algorithm, and its optimization process is shown in Figure 3.

\section{Numerical Simulation}

3.1. Modeling of Bridge. Simply supported bridge with rectangular cross-section as shown in Figure 4 is adopted as numerical model to verify effectiveness of the proposed method. The length $L$ is $20 \mathrm{~m}$, the sectional width $B$ is $0.8 \mathrm{~m}$, and height 


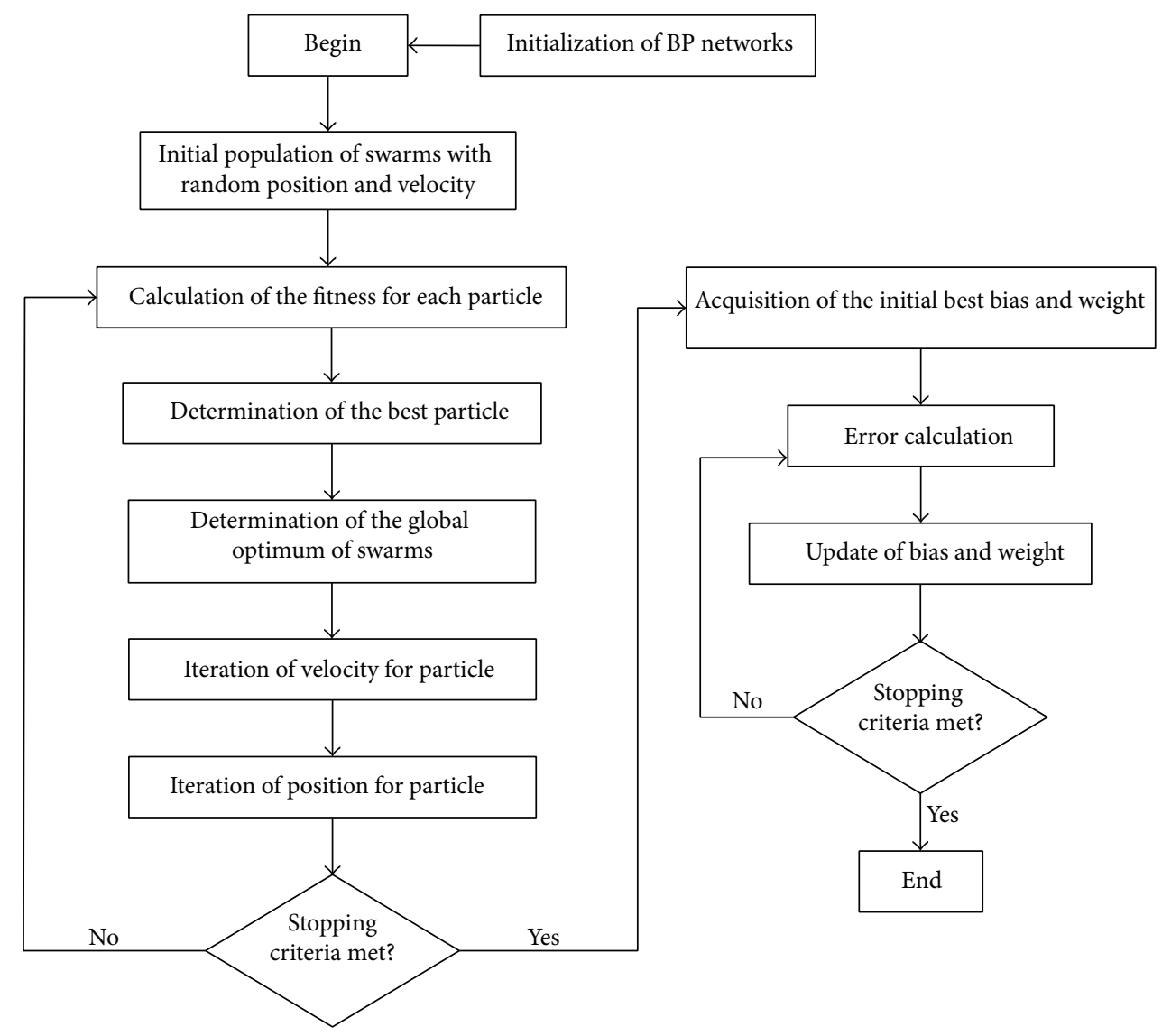

FIgURE 3: Flowchart of ANNs optimized by PSO.

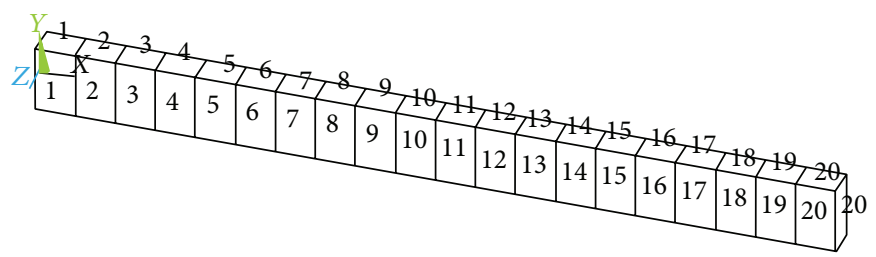

FIgURE 4: Simply supported beam bridge.

$H$ is $1.7 \mathrm{~m}$. The model is divided into 20 elements, the elastic modulus $E=3.25 e 10 \mathrm{~N} / \mathrm{m}^{2}$, Poisson's ratio $\mu=0.167$, and the material density $\rho=2600 \mathrm{~kg} / \mathrm{m}^{3}$.

In numerical simulation, damage of structure is represented by reduction in element stiffness. In this paper, the identifications with single damage location and multiple locations are conducted, respectively. As for damage identification with single location, stiffness of element 10 is assumed to drop $5 \%, 10 \%, 15 \%$, and $20 \%$. As for the multiple one, stiffnesses of elements 5 and 8 drop 5\%,10\%, 15\%, and 20\% simultaneously. Natural frequencies and mode shapes for damaged and intact structure can be obtained through Lanczos modal analysis method, and the first four vertical bending modes are calculated. Natural frequencies for undamaged structure are listed in Table 1.
TABLE 1: Natural frequencies for undamaged structure.

\begin{tabular}{lcccc}
\hline Modal order & 1 & 2 & 3 & 4 \\
\hline Natural frequencies $(\mathrm{Hz})$ & 6.7982 & 26.955 & 59.788 & 104.26 \\
\hline
\end{tabular}

3.2. Damage Localization Based on Modal Flexibility Indicators. The modal flexibility matrices for structure before and after damage can be calculated by natural frequencies and mode shapes according to (1). And modal flexibility changes can be obtained by (2). The maximum absolute values of elements in each column of matrices of modal flexibility changes are displayed for damage localization. In order to illustrate the relationship between number of modes and identification effects, modal flexibilities are calculated by the first order mode and first four-order modes, respectively. 


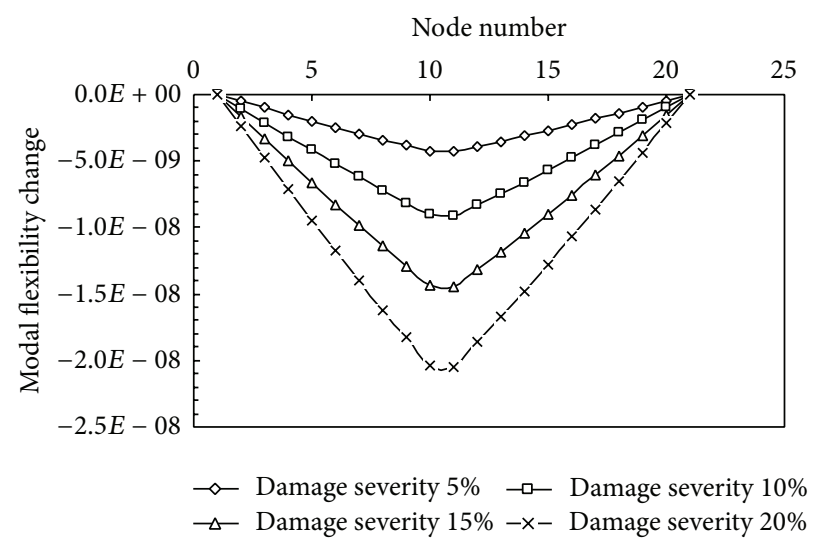

(a) Single damage location (element 10)

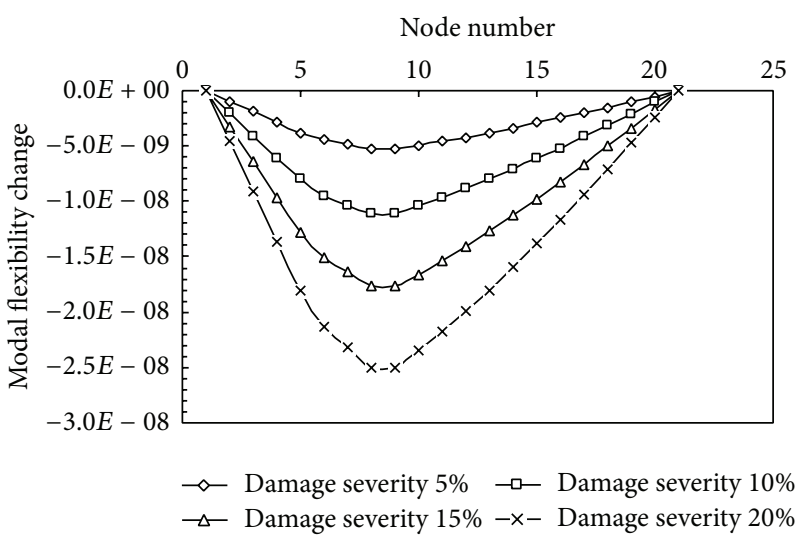

(b) Multiple damage locations (elements 5 and 8)

Figure 5: Damage localization based on modal flexibility changes using the first order mode.

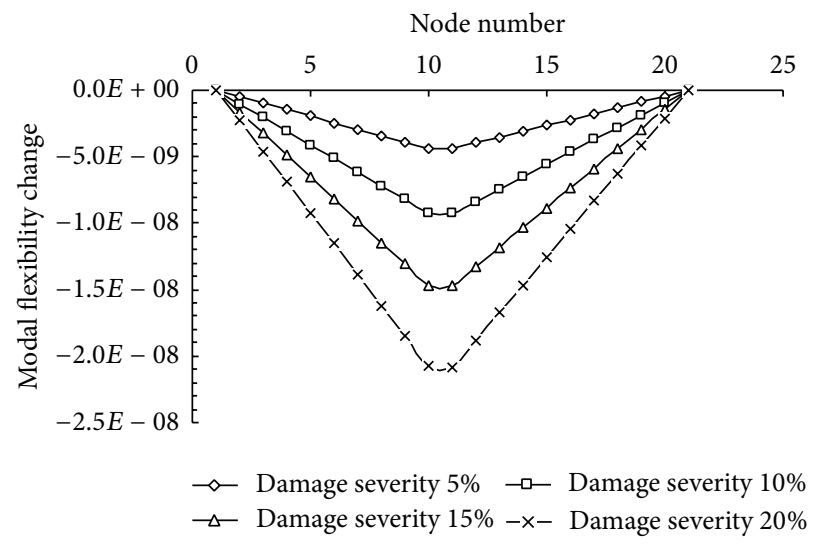

(a) Single damage location (element 10)

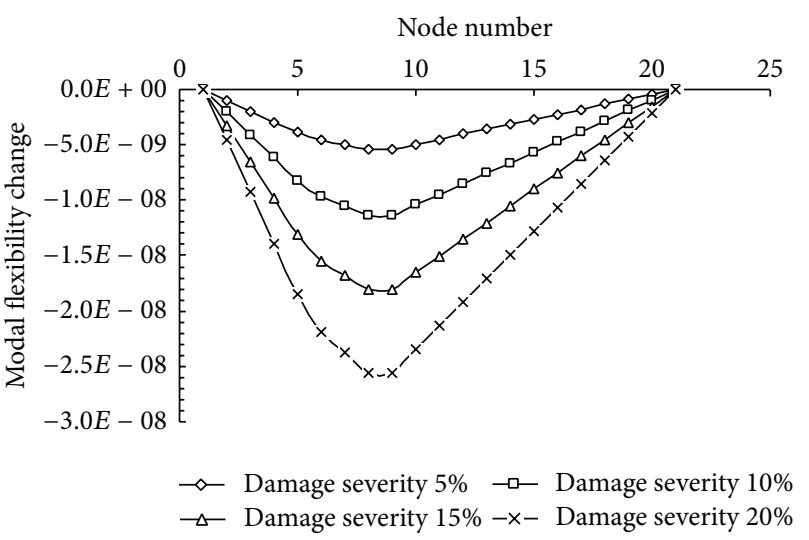

(b) Multiple damage locations (elements 5 and 8 )

Figure 6: Damage localization based on modal flexibility changes using the first four-order modes.

3.2.1. Modal Flexibility Changes. The damage localization results of modal flexibility changes using the first order mode are shown in Figure 5.

Damage localization results of modal flexibility changes using the first four-order modes are shown in Figure 6.

As can be seen from Figures 5 and 6, modal flexibility changes can identify the damage presence but cannot be able to identify the accurate damage locations. Curves calculated by the first order mode are consistent with that calculated by the first four-order modes.

3.2.2. Curvature of Modal Flexibility Changes. Damage localization results for curvature of modal flexibility changes using the first order mode are shown in Figure 7.

Damage localization results for curvature of modal flexibility changes using the first four-order modes are shown in Figure 8.

As can be seen from Figures 7 and 8, curvature of modal flexibility changes calculated by the first order mode and the first four-order modes can identify the damage locations and also qualitatively determine the damage severity. Curvature curves obtained by the first four-order modes are smoother than that by the first order mode, and oscillation at undamaged locations is smaller. However, it is sufficient for damage localization based on the curvature calculated by the first order mode, and it is more feasible in practical applications.

\subsection{Damage Severity Identification Based on Neural Network Optimized by PSO}

3.3.1. Determination of Damage Indicator. Considering the simplicity of modal flexibility changes, the normalized vectors are used as damage indicators and inputs of neural networks optimized by PSO. The normalized vector can be calculated by

$$
\begin{aligned}
\text { Input } & =\left\{\overline{\delta_{1}}, \overline{\delta_{2}}, \ldots, \overline{\delta_{n}}\right\}, \\
\overline{\delta_{j}} & =\frac{\delta_{j}-\delta_{\min }}{\delta_{\max }-\delta_{\min }},
\end{aligned}
$$

where $\delta_{j}$ is the maximum absolute value of elements in $j$ th column of $\Delta F ; \delta_{\max }$ and $\delta_{\min }$ are maximum and minimum 


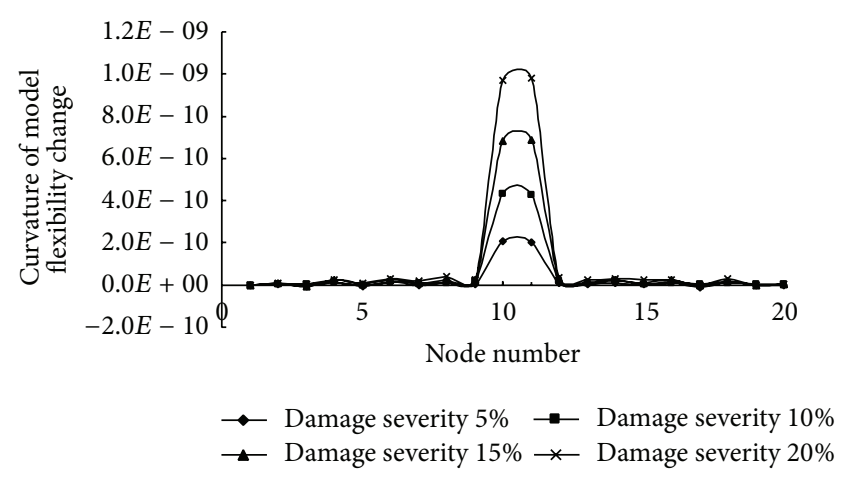

(a) Single damage location (element 10)

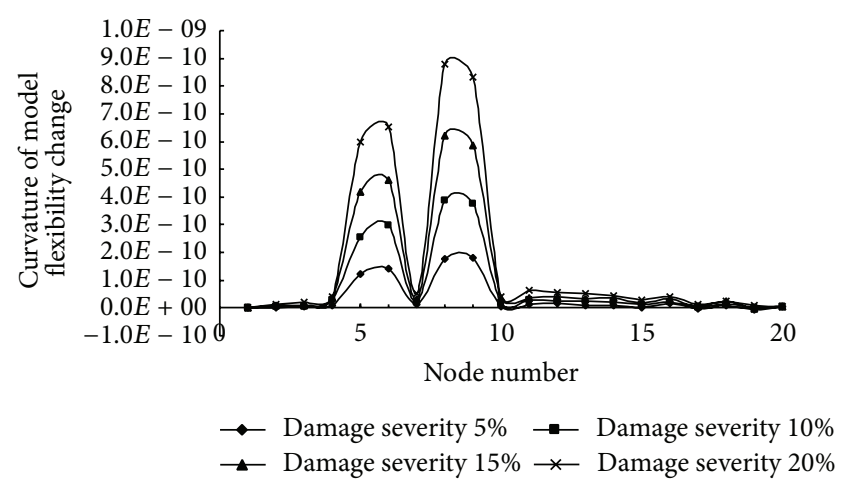

(b) Multiple damage locations (elements 5 and 8 )

FIGURE 7: Damage localization based on curvature of modal flexibility changes using the first order mode.

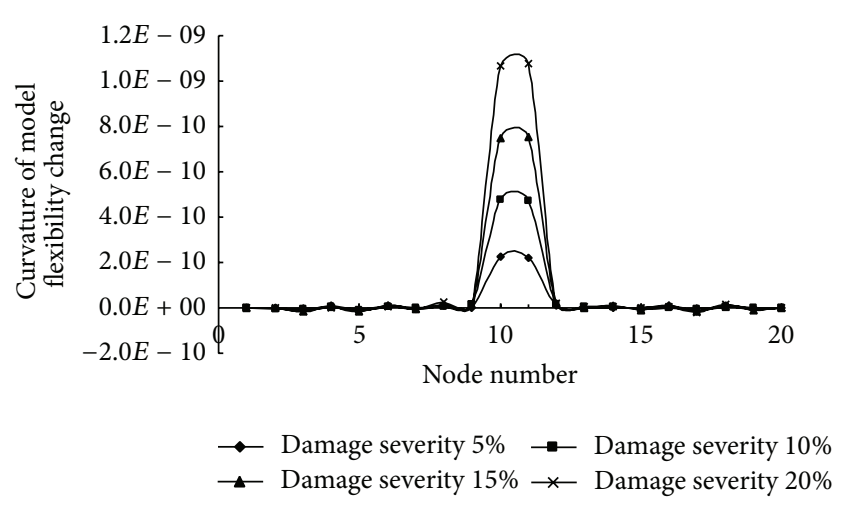

(a) Single damage location (element 10)

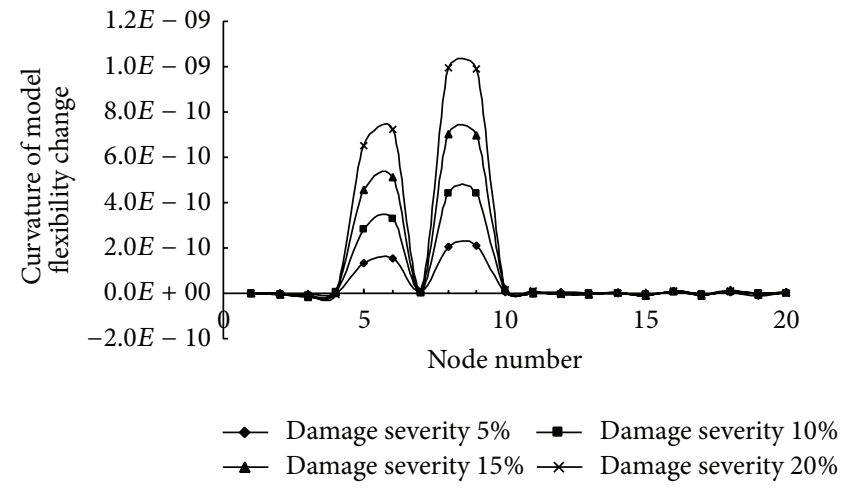

(b) Multiple damage locations (elements 5 and 8)

FIGURE 8: Damage localization based on curvature of modal flexibility changes using the first four-order modes.

values of vector $\delta(j=1,2, \ldots, n)$, respectively; $\overline{\delta_{j}}$ is the normalized $\delta_{j}$.

\subsubsection{Severity Identification with Single Damage Location.} Taking the damage identification of element 10 , for example, damage severities with $5 \%, 10 \%, 15 \%$, and $20 \%$ are selected as training samples, while $7 \%, 12 \%$, and $18 \%$ are testing ones. The normalized vectors of modal flexibility changes are used as input variables of neural network optimized by PSO. The training samples for damage identification are listed in Table 2.

Testing samples are used to verify the feasibility of optimized neural network, and the identification results are listed in Table 3.

As can be seen from Table 3, the maximum relative errors for neural network optimized by PSO are $2.57 \%$. It reveals that the identification accuracy is favorable and can meet the practical requirements. The neural network optimized by PSO is suitable for damage severity identification with single damage location of bridge.

3.3.3. Severity Identification with Multiple Damage Locations. Taking damage identification of elements 5 and 8 , for example, damage severities simultaneously with $5 \%, 10 \%$, $15 \%$, and $20 \%$ are used as training samples, and simultaneously $7 \%, 12 \%$, and $18 \%$ are treated as testing ones. The corresponding training samples are listed in Table 4 , and the identification results for testing samples are shown in Table 5.

As can be seen from Table 5, the maximum relative error is $6.22 \%$. It is larger than that of identification with single damage location. The reasons are that it is more complex for the severities identification with multiple damage locations and also associated with the number and rationality of training samples.

3.3.4. Comparative Analysis with Traditional BP Neural Network. In order to verify the superiority of the proposed method, comparative analysis between PSO-BP and traditional network is conducted. The identification results for severity identification with single and multiple damage locations are listed in Table 6.

As can be seen from Table 6 , the maximum relative errors of BP neural network for severities identification with single and multiple locations are $4.00 \%$ and $12.00 \%$, respectively. Comparative results show that the calculation accuracy of neural network optimized by PSO is better than traditional BP network. 
TABLE 2: Training samples for damage severity identification of element 10.

\begin{tabular}{lcccc}
\hline Node number & 5 & \multicolumn{3}{c}{ Damage severity (\%) } \\
\hline 2 & 5 & 10 & 15 & 20 \\
3 & 0.01 & 0.01 & 0.01 & 0.01 \\
4 & 0.14 & 0.14 & 0.14 & 0.14 \\
5 & 0.27 & 0.27 & 0.27 & 0.27 \\
6 & 0.40 & 0.40 & 0.40 & 0.40 \\
7 & 0.52 & 0.52 & 0.52 & 0.52 \\
8 & 0.64 & 0.64 & 0.64 & 0.64 \\
9 & 0.77 & 0.76 & 0.76 & 0.76 \\
10 & 0.88 & 0.88 & 0.88 & 0.88 \\
11 & 0.99 & 0.99 & 0.99 & 0.99 \\
12 & 1.00 & 1.00 & 1.00 & 1.00 \\
13 & 0.90 & 0.90 & 0.90 & 0.90 \\
14 & 0.80 & 0.80 & 0.80 & 0.80 \\
15 & 0.69 & 0.69 & 0.69 & 0.69 \\
16 & 0.58 & 0.58 & 0.58 & 0.58 \\
17 & 0.47 & 0.47 & 0.47 & 0.47 \\
18 & 0.35 & 0.35 & 0.35 & 0.35 \\
19 & 0.24 & 0.24 & 0.24 & 0.24 \\
20 & 0.12 & 0.12 & 0.12 & 0.12 \\
\hline & 0.00 & 0.00 & 0.00 & 0.00 \\
\hline
\end{tabular}

TABLE 3: Damage severity identification results for testing samples of element 10 .

\begin{tabular}{lcc}
\hline $\begin{array}{l}\text { Expected outputs } \\
(\%)\end{array}$ & $\begin{array}{c}\text { Identification results } \\
(\%)\end{array}$ & $\begin{array}{c}\text { Relative errors } \\
(\%)\end{array}$ \\
\hline 7 & 7.18 & 2.57 \\
12 & 12.28 & 2.28 \\
18 & 18.35 & 1.94 \\
\hline
\end{tabular}

\section{Conclusions}

Damage identification is one of the major challenges in bridge health monitoring. We have proposed a two-stage strategy based on modal flexibility and neural network optimized by PSO for damage location and severity identification. Simply supported bridge with rectangular cross-section is selected as numerical model to verify the effectiveness of the proposed method.

As for damage localization based on modal flexibility indicators, modal flexibility changes can identify the damage presence but cannot achieve acceptable identification of damage locations. The curvature of flexibility changes is more favorable for damage localizing and it is sufficient to be calculated by the first order mode.

With regard to the damage severity identification of neural network optimized by PSO, normalized vector of modal flexibility changes is adopted as input variable. Training samples for identification with single and multiple damage locations are constructed, respectively. The identification results for single damage location reveal that the maximum relative error is $2.57 \%$, while it is $6.22 \%$ for multiple damage
TABLE 4: Training samples for damage severity identification of elements 5 and 8 .

\begin{tabular}{lcccc}
\hline \multirow{2}{*}{ Node number } & 5 & \multicolumn{3}{c}{ Damage severity $(\%)$} \\
\hline 2 & 5 & 10 & 15 & 20 \\
3 & 0.10 & 0.10 & 0.10 & 0.10 \\
4 & 0.30 & 0.30 & 0.30 & 0.30 \\
5 & 0.50 & 0.50 & 0.50 & 0.50 \\
6 & 0.69 & 0.69 & 0.69 & 0.69 \\
7 & 0.83 & 0.83 & 0.83 & 0.83 \\
8 & 0.92 & 0.92 & 0.92 & 0.92 \\
9 & 1.00 & 1.00 & 1.00 & 1.00 \\
10 & 1.00 & 1.00 & 1.00 & 1.00 \\
11 & 0.93 & 0.93 & 0.93 & 0.93 \\
12 & 0.85 & 0.85 & 0.85 & 0.85 \\
13 & 0.78 & 0.77 & 0.77 & 0.77 \\
14 & 0.69 & 0.69 & 0.69 & 0.69 \\
15 & 0.60 & 0.60 & 0.60 & 0.60 \\
16 & 0.50 & 0.51 & 0.51 & 0.51 \\
17 & 0.41 & 0.41 & 0.41 & 0.41 \\
18 & 0.31 & 0.31 & 0.31 & 0.31 \\
19 & 0.21 & 0.21 & 0.21 & 0.21 \\
20 & 0.10 & 0.10 & 0.10 & 0.10 \\
\hline & 0.00 & 0.00 & 0.00 & 0.00 \\
\hline
\end{tabular}

TABle 5: Damage severity identification results for testing samples of elements 5 and 8 .

\begin{tabular}{lcc}
\hline $\begin{array}{l}\text { Expected outputs } \\
(\%)\end{array}$ & $\begin{array}{c}\text { Identification results } \\
(\%)\end{array}$ & $\begin{array}{c}\text { Relative errors } \\
(\%)\end{array}$ \\
\hline 7,7 & $6.57,7.35$ & $6.14,5.00$ \\
12,12 & $11.38,12.68$ & $5.17,5.67$ \\
18,18 & $19.12,18.95$ & $6.22,5.28$ \\
\hline
\end{tabular}

TABle 6: Damage identification using BP network.

\begin{tabular}{lcc}
\hline Damage category & $\begin{array}{c}\text { Single damage } \\
\text { location }\end{array}$ & $\begin{array}{c}\text { Multiple damage } \\
\text { locations }\end{array}$ \\
\hline $\begin{array}{l}\text { Expected outputs } \\
(\%)\end{array}$ & 7 & 7,7 \\
& 12 & 12,12 \\
Actual results & 78 & 18,18 \\
$(\%)$ & 12.38 & $7.84,7.79$ \\
& 17.59 & $11.13,12.89$ \\
Relative errors & 4.00 & $16.83,16.98$ \\
$(\%)$ & 3.17 & $12.00,11.29$ \\
& 2.28 & $7.25,7.42$ \\
\hline
\end{tabular}

locations. The results manifest that it is feasible for damage severity identification using neural network optimized by PSO. Comparative analysis with traditional BP network is conducted to verify its superiority. The calculation accuracy of optimized neural network is more favorable. 


\section{Conflict of Interests}

The authors declare that there is no conflict of interests regarding the publication of this paper.

\section{Acknowledgments}

The authors express their appreciation for the financial support of National Natural Science Foundation of China under Grant nos. 51378236 and 51278222 and "985 Project" of Jilin University.

\section{References}

[1] N. Kulprapha and P. Warnitchai, "Structural health monitoring of continuous prestressed concrete bridges using ambient thermal responses," Engineering Structures, vol. 40, pp. 20-38, 2012.

[2] J. Kim, J. Park, and B. Lee, "Vibration-based damage monitoring in model plate-girder bridges under uncertain temperature conditions," Engineering Structures, vol. 29, no. 7, pp. 1354-1365, 2007.

[3] S. C. Mohan, D. K. Maiti, and D. Maity, "Structural damage assessment using FRF employing particle swarm optimization," Applied Mathematics and Computation, vol. 219, no. 20, pp. 10387-10400, 2013.

[4] U. Dackermann, Vibration-based damage identification methods for civil engineering structures using artificial neural networks [Ph.D. thesis], Faculty of Engineering and Information Technology, University of Technology Sydney, Sydney, Australia, 2010.

[5] B. H. Kim, T. Park, and G. Z. Voyiadjis, "Damage estimation on beam-like structures using the multi-resolution analysis," International Journal of Solids and Structures, vol. 43, no. 14-15, pp. 4238-4257, 2006.

[6] S. W. Doebling, C. R. Farrar, and M. B. Prime, "A summary review of vibration-based damage identification methods," Shock and Vibration Digest, vol. 30, no. 2, pp. 91-105, 1998.

[7] Y. J. Yan, L. Cheng, Z. Y. Wu, and L. H. Yam, "Development in vibration-based structural damage detection technique," Mechanical Systems and Signal Processing, vol. 21, no. 5, pp. 2198-2211, 2007.

[8] P. Cawley and R. D. Adams, "The location of defects in structures from measurements of natural frequencies," The Journal of Strain Analysis for Engineering Design, vol. 14, no. 2, pp. 4957, 1979.

[9] A. K. Pandey and M. Biswas, "Damage detection in structures using changes in flexibility," Journal of Sound and Vibration, vol. 169, no. 1, pp. 3-17, 1994.

[10] G. Li, K. Hao, Y. Lu, and S. Chen, "A flexibility approach for damage identification of cantilever-type structures with bending and shear deformation," Computers and Structures, vol. 73, no. 6, pp. 565-572, 1999.

[11] L. T. Stutz, D. A. Castello, and F. A. Rochinha, "A flexibilitybased continuum damage identification approach," Journal of Sound and Vibration, vol. 279, no. 3-5, pp. 641-647, 2005.

[12] E. Reynders and G. de Roeck, "A local flexibility method for vibration-based damage localization and quantification," Journal of Sound and Vibration, vol. 329, no. 12, pp. 2367-2383, 2010.

[13] F. N. Catbas, M. Gul, and J. L. Burkett, "Damage assessment using flexibility and flexibility-based curvature for structural health monitoring," Smart Materials and Structures, vol. 17, no. 1, pp. 1-12, 2008.
[14] J. J. Lee, J. W. Lee, J. H. Yi, C. B. Yun, and H. Y. Jung, "Neural networks-based damage detection for bridges considering errors in baseline finite element models," Journal of Sound and Vibration, vol. 280, no. 3-5, pp. 555-578, 2005.

[15] M. Vakil-Baghmisheh, M. Peimani, M. H. Sadeghi, and M. M. Ettefagh, "Crack detection in beam-like structures using genetic algorithms," Applied Soft Computing Journal, vol. 8, no. 2, pp. 1150-1160, 2008.

[16] F. Kang, J. Li, and Q. Xu, "Damage detection based on improved particle swarm optimization using vibration data," Applied Soft Computing Journal, vol. 12, no. 8, pp. 2329-2335, 2012.

[17] J. Min, S. Park, C. Yun, C. Lee, and C. Lee, "Impedance-based structural health monitoring incorporating neural network technique for identification of damage type and severity," Engineering Structures, vol. 39, pp. 210-220, 2012.

[18] Y. B. Jiao, H. B. Liu, Y. C. Cheng, and X. Q. Wang, "Fuzzy neural network-based damage assessment of bridge under temperature effect," Mathematical Problems in Engineering, vol. 2014, Article ID 418040, 9 pages, 2014.

[19] M. Mehrjoo, N. Khaji, H. Moharrami, and A. Bahreininejad, "Damage detection of truss bridge joints using Artificial Neural Networks," Expert Systems with Applications, vol. 35, no. 3, pp. 1122-1131, 2008.

[20] R. Ganguli, "A fuzzy logic system for ground based structural health monitoring of a helicopter rotor using modal data," Journal of Intelligent Material Systems and Structures, vol. 12, no. 6, pp. 397-407, 2001.

[21] J. Kennedy and R. Eberhart, "Particle swarm optimization," in Proceedings of the IEEE International Conference on Neural Networks, pp. 1942-1948, December 1995.

[22] M. A. Mohandes, "Modeling global solar radiation using Particle Swarm Optimization (PSO)," Solar Energy, vol. 86, no. 11, pp. 3137-3145, 2012.

[23] M. Sharafi and T. Y. Elmekkawy, "Multi-objective optimal design of hybrid renewable energy systems using PSO-simulation based approach," Renewable Energy, vol. 68, pp. 67-79, 2014.

[24] J. Q. Chen, Y. F. Tang, R. Ge, Q. L. An, and X. W. Guo, "Reliability design optimization of composite structures based on PSO together with FEA," Chinese Journal of Aeronautics, vol. 26, no. 2, pp. 343-349, 2013.

[25] S. Kazemi, A. Fooladi, and A. R. Rahai, "Implementation of the modal flexibility variation to fault identification in thin plates," Acta Astronautica, vol. 66, no. 3-4, pp. 414-426, 2010.

[26] O. Begambre and J. E. Laier, "A hybrid Particle Swarm Optimization-Simplex algorithm (PSOS) for structural damage identification," Advances in Engineering Software, vol. 40, no. 9, pp. 883-891, 2009.

[27] A. Khare and S. Rangnekar, "A review of particle swarm optimization and its applications," Applied Soft Computing, vol. 13, pp. 2997-3006, 2013.

[28] H. S. Wang, Y. N. Wang, and Y. C. Wang, "Cost estimation of plastic injection molding parts through integration of PSO and BP neural network," Expert Systems with Applications, vol. 40, no. 2, pp. 418-428, 2013. 


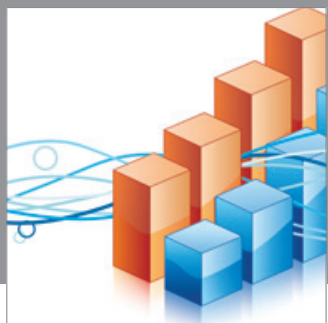

Advances in

Operations Research

mansans

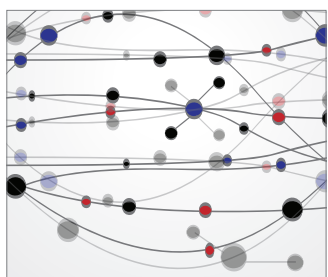

The Scientific World Journal
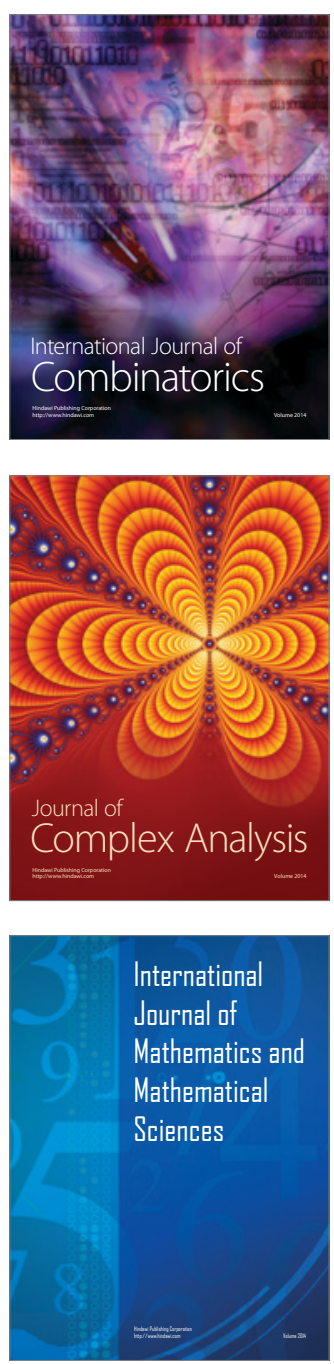
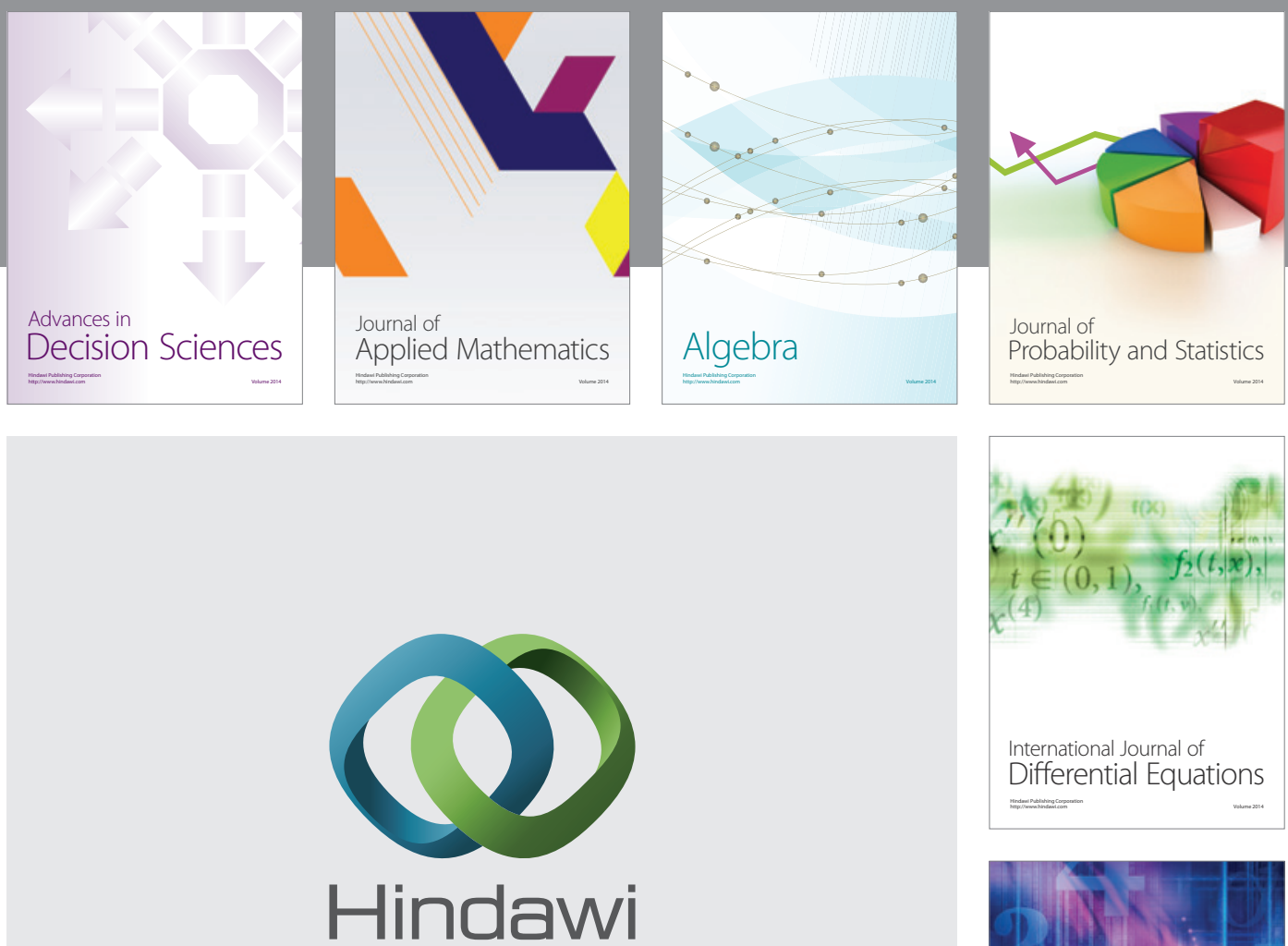

Submit your manuscripts at http://www.hindawi.com
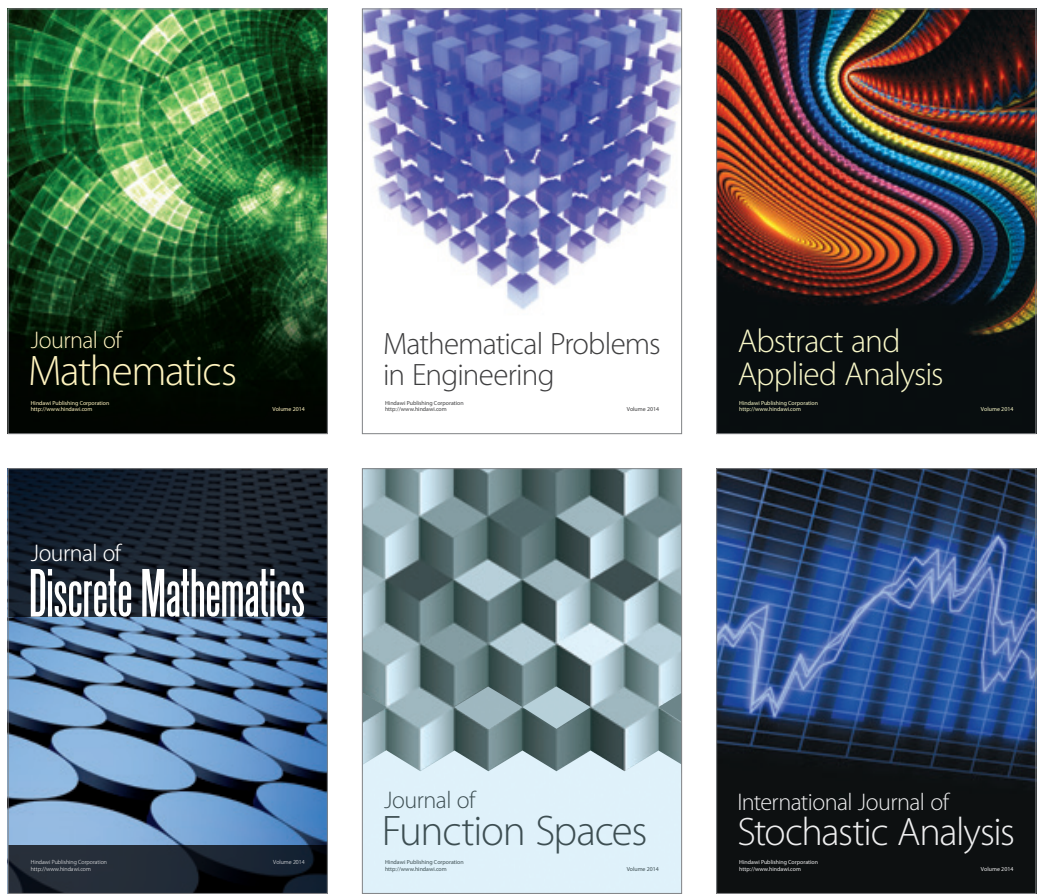

Journal of

Function Spaces

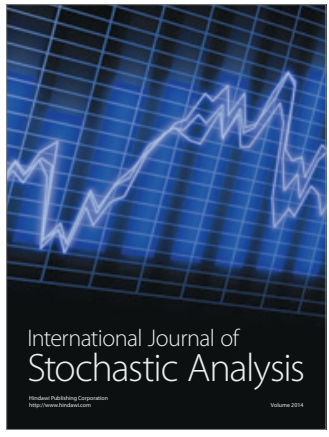

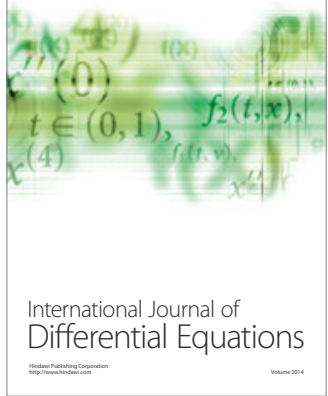
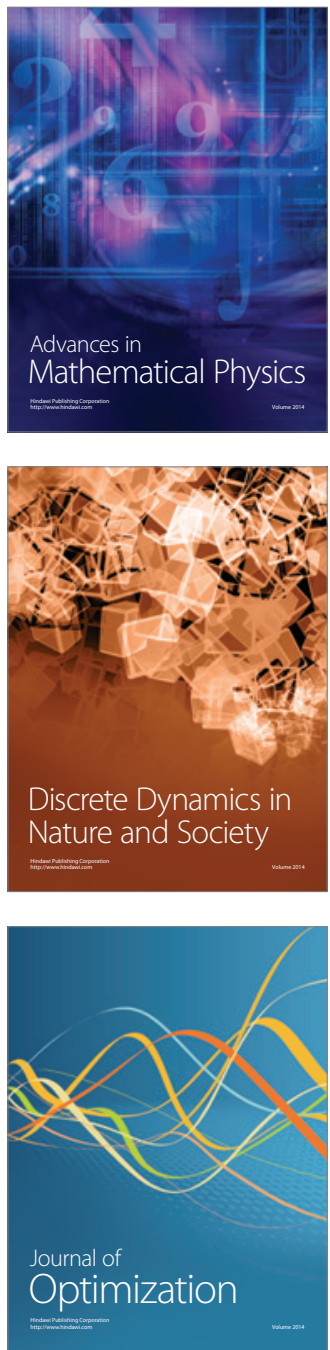